\title{
Multi-wavelength raman characterization of back-gated monolayer and bilayer Graphene
}

\author{
Maedeh Arvani ${ }^{1, *}$, Mahdi Aghajanloo ${ }^{2}$ \\ ${ }^{1}$ Tampere University of Technology, Korkeakoulunkatu 10, 33720 Tampere, Finland \\ ${ }^{2}$ Halmstad University, Kiristian Vag 3, 30118, Halmstad, Sweden \\ Email address: \\ Maedeh.Arvani@tut.fi (M. Arvani),mahdiag@student.chalmers.se (M. Aghajanloo) \\ To cite this article: \\ Maedeh Arvani, Mahdi Aghajanloo. Multi-Wavelength Raman Characterization of Back-Gated Monolayer and Bilayer Graphene. \\ American Journal of Modern Physics. Vol. 3, No. 4, 2014, pp. 168-172. doi: 10.11648/j.ajmp.20140304.13
}

\begin{abstract}
In this work, we investigate the Raman spectrum of gated monolayer and bilayer graphene devices. We used Raman spectroscopy with three different excitation wavelengths: (488nm, $514 \mathrm{~nm}$ and $633 \mathrm{~nm}$ ). After producing graphene sheets by scotch tape technique, Raman spectrometry used to distinguish between bilayer, mono layer and other few layer of graphene. We contact the wires on the flakes using micro-soldering method then we applied gate voltage on monolayer and bilayer graphene and investigate the changes in peak of the Raman spectra in different wavelengths in different voltages. Raman spectroscopy probes phonons as well as electronic states. If the electronic dispersion changes, the Raman spectrum will also changes. The shifts of the Raman spectra peaks of the monolayer and bilayer are explained in the current work. Charge carrier concentration as a function of gate voltage in gated graphene is shown as well as the position of the $\mathrm{G}$ peak and 2D peak graphene versus gate voltage. For monolayer devices we observed the expected behavior for doped devices. For bilayer devices, we present a comparison between the theoretical model and our experimental results.
\end{abstract}

Keywords: Monolayer Graphene, Bilayer Graphene, Raman Spectroscopy, Nanoelectronics

\section{Introduction}

Graphene is an allotrope of carbon. It has a two dimensional crystal structure, that is, just one-atom thick. The atoms are arranged tightly in a honeycomb lattice. Graphene is considered the basic building form of all other allotropes of carbon. Graphite can be thought as stacked sheets of graphene one on top of each other, nanotubes are sheets of graphene which are rolled and fullerenes are wrapped sheets of graphene [1].The length of the bond between two atoms of carbon is approximately $0.142 \mathrm{~nm}$, and the distance between two sheets of graphene in graphite is $0.335 \mathrm{~nm}$. Raman spectroscopy has proved an invaluable tool in graphene research. It can unambiguously and non-destructively determines its monolayer structure, determined by the shape of the 2D peak. Furthermore, it can provide insights regarding the doping level, the amount of disorder and the edge geometry [2]. Due to its special electronic behavior and its structural properties, it seems that graphene surpasses many metals and other conventional semiconductors. The Raman spectra of graphene show features in $800-2000 \mathrm{~cm}^{-1}$ [2]. The $\mathrm{G}$ peak, which corresponds to the $\mathrm{E} 2 \mathrm{~g}$ phonon at the central zone of the Brillouin zone, lies at $1580 \mathrm{~cm}^{-1}$. The D peak, which is a breathing mode for sp2 atoms, is a defect activated peak. Usually, as there are not enough structural defects in pristine graphene, the D peak is not present. It can however be found at the edges of flakes. The second order of the D peak, the so-called $2 \mathrm{D}$ peak, is always present, even when there are no defects. The 2D peak lies at $2700 \mathrm{~cm}^{-1}$ [2].The shape of the 2D peak gives us information about the number of layers. Monolayer graphene has a sharp 2D peak, while few-layers and thick graphite displays a broader 2D peak which consists of several unresolved peaks. Fig 1 shows the Raman spectrum of graphene and few-layer graphene. This fig shows that the 2D peak is a single sharp peak in graphene, while in graphite it contains a shoulder. 


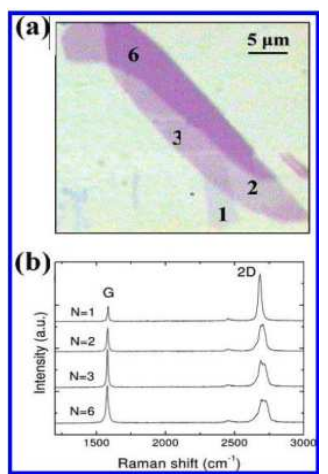

Fig. 1. Evolution of the Raman spectrum of graphene as a function of layer thickness. Monolayer graphene has a single sharp peak [3]

The $\mathrm{G}$ peak position of undoped graphene is at $1580 \mathrm{~cm}^{-1}$ and for increasing doping the position increases. The $G$ shifting is due to the non-adiabatic removal of the Kohn anomaly at $\Gamma$ [4].The Kohn anomaly is an anomaly, which describes the relation of a phonon branch in a metal.

\section{Experiment}

\subsection{Fabrication of Graphene (Scotch Tape Technique)}

The experimental part of the thesis project has been down in the Freie University of Berlin in AG Casiraghi's laboratory. In this thesis we used the method originally used to isolate graphene. A piece of graphite is peeled off by scotch tape and then the flakes are transferred onto a substrate. Graphene was deposited on a silicon substrate covered with a layer of silicon oxide. This process is called micromechanical cleavage (the Scotch-tape method as it has been colloquially known). This is used to isolated graphene to provide optical enhancement that makes graphene visible under the optical microscope. The silicon, which is under $\mathrm{SiO}^{2}$, can be used as a "back gate" electrode.

By this method, we can fabricate pristine graphene. Graphene can also be transferred so called wedging technique [5].The procedure is as follows: the graphene flake is first encapsulated in a polymer. Then the sample is immersed in deionized water. The water intercalates at the substrate-polymer interface, since the former is hydrophilic and the latter hydrophobic. This causes the graphene flakes to be lifted off the substrate, while still attached to the polymer film. Subsequently, the graphene encapsulated polymer is deposited onto a new piece of substrate the sample is then heated to $70{ }^{\circ} \mathrm{C}$ in order to evaporate any water residuals on the surface. The results show that the doping can be removed by transferring the flakes on a clean silicon substrate. This could indicate that the doping is related to the oxygen and/or water trapped at the silicon surface after plasma cleaning. However it is also possible that the polymer used to encapsulate graphene could dope graphene, compensating the existing charges. Our substrate consists of 550-micron thick silicon covered with $90 \mathrm{~nm}$ of thermally grown silicon oxide.

Method for contacting nanoscale films e.g. graphene is submicron soldering. We used this method to fabricate a gate contact to the graphene to be able to apply a high electric field between the graphene and the silicon back contact. This kind of samples allowed us to carry out Raman spectroscopy of gated pristine monolayer- as well as bi-layer graphene. The submicron soldering set up consists of an optical microscope, a sample heater and micromanipulator [6].

\subsection{Identification of Monolayer and Bilayer Graphene}

The first step in working with graphene or its bilayer is identifying it on a substrate using a microscope. Our graphene monolayer and bilayer samples are provided by Dr. Casiraghi's group at the University of Manchester. We selected only flakes that look thin (based on their color). Afterwards, we measured the Raman spectrum. From Fig 3.2 , we observe that the shape of the $2 \mathrm{D}$ peak is a single, sharp Lorentzian peak. This is characteristic for monolayer graphene. Fig 2 shows an optical micrograph of a graphene flake attached to a bilayer and few-layer. Graphene is the light violet part.

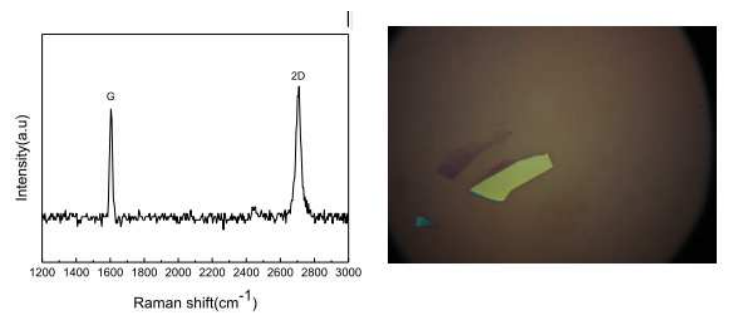

Fig. 2. Raman spectra of monolayer graphene (left), An optical micrograph of a graphene sample (Right).

Fig 3 shows bilayer graphene, as determined by the shape of the $2 \mathrm{D}$ peak.

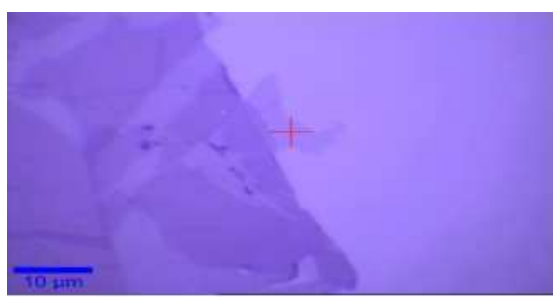

Fig. 3. Bilayer Graphene.

However, we can see a split in the G peak of the bilayer, In order to investigate the nature of this splitting; we applied a gate voltage to the sample. We applied a gate voltage to the monolayer and bilayer graphene and the positions of the $G$ and 2D peaks versus gate voltage were recorded under different wavelength. The $\mathrm{G}$ peak in bilayer graphene shows a single $G$ component with a spectral position similar to monolayer graphene. When we apply gate voltage, due to the external electric field, the Fermi level position will change. The changes in the Fermi level make charge transfer split the $\mathrm{G}$ peak for the $\mathrm{E} 2 \mathrm{~g}$ phonon mode. This mode shows very strong coupling between electrons and phonons [7]. The Raman $G$ peak will have two components, which are associated with symmetric (S) and asymmetric (AS) 
vibration of the atoms in the two sheets of bilayer graphene. In the bilayer, usually a single peak is detected that can split if the two sheets are doped differently. When we have a doped bilayer, we can find two components in the G peak because the symmetry is broken by doping due to different $n$ top and $n$ bottom [7]. The self-energy of the S and AS phonons for different Fermi energies is calculated by Ando. He found a hardening of the symmetric and asymmetric phonons, strongly influenced by carrier doping [7].

In this work, our experimental results confirm the theory and we can see that the $G$ peak of the bilayer graphene at around $1580 \mathrm{~cm}-1$ has two components that exhibit opposite dependence as the Fermi level of energy is tuned. The results are in agreement with Ando calculations. The splitting of the $\mathrm{G}$ peak in bilayer graphene can be used to find the electron concentration, $\mathrm{n}$.

\subsection{Charge Concentration in Gated Graphene}

The gate voltage changes the Fermi energy $\varepsilon \mathrm{f}$ and the Fermi surface of graphene. The following equation relates the gate voltage to amount of charge:

$$
\mathrm{n}=\eta\left(\mathrm{V}_{\mathrm{g}}-\mathrm{V}_{\mathrm{n}}\right)
$$

Where $\mathrm{V}_{\mathrm{g}}$ is applied gate voltage, $\mathrm{V}_{\mathrm{n}}$ is the gate voltage required to reach the charge neutrality point, and $\eta$ is the capacitive coupling $\eta$ can be calculated as:

$$
\eta=\left(\varepsilon_{\mathrm{r}} \cdot \varepsilon_{0}\right) /\left(\mathrm{t}_{\mathrm{ox}} \mathrm{e}\right)
$$

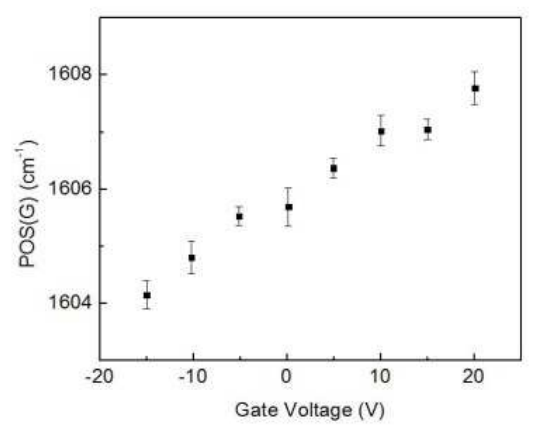

a)

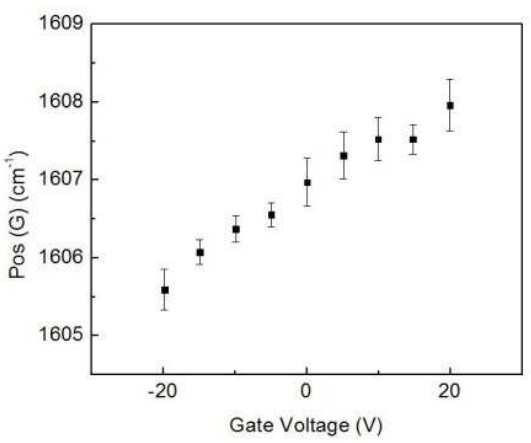

c)
Where $\varepsilon 0$ is vacuum permittivity, $\varepsilon \mathrm{r}$ is permittivity of silicon; $t$ ox is the thickness of the silicon oxide and $e$ is electron charge. In our case, $\varepsilon_{\mathrm{r}}=3.9$ and $\varepsilon_{0}=8.85^{*} 10^{-12} \mathrm{Fm}^{-1}$ and $\mathrm{t}$ ox $=90 \mathrm{~nm}$ therefore $\eta=2.39 * 10^{\wedge} 11 \mathrm{~cm}^{-1} \mathrm{~V}^{-1}$. If the sample is undoped, at $V_{n}=0$ we have $n=\eta \cdot V_{g}$. When the sample is doped (i.e. the G peak position $>1582 \mathrm{~cm}^{-1}$ ) then we have to add an extra voltage to have graphene at the charge neutrality point.

\section{Results}

\subsection{Gated Monolayer Graphene}

When we apply gate voltage the Fermi level will change. This results in changes in the positions of the $\mathrm{G}$ peak and the $2 \mathrm{D}$ peak. We measure $\mathrm{G}$ peak and $2 \mathrm{D}$ peak shifts versus gate voltage from-30V up to $+30 \mathrm{~V}$ by voltage step $1 \mathrm{~V}$ at three different wavelengths (633nm, 514nm and 488nm). Fig 4 shows $G$ peak and 2D peak shift versus gate voltage from $-20 \mathrm{~V}$ up to $+20 \mathrm{~V}$. Our data show a linear relationship between gate voltage and $G$ peak position, and similar relationship between gate voltage and $2 \mathrm{D}$ peak position. As we can see in Fig 4.1, for both wavelengths, the position of the $G$ peak shifts to higher energy for increasing whole doping and to lower energy for electron doping. Fig 5 shows the $2 \mathrm{D}$ peak position.

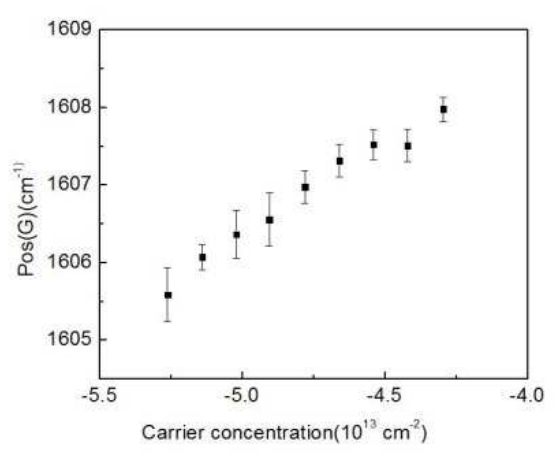

b)

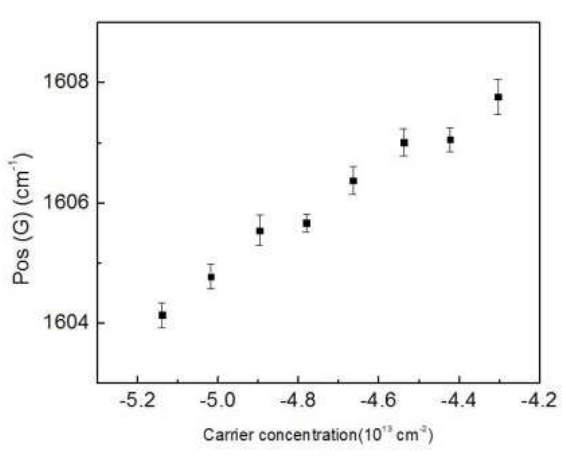

d)

Fig. 4. $\operatorname{POS}(G)$ as a function of : a) applied gate voltage $\lambda=633$, b) carrier concentration, $\lambda=633$ c) applied gate voltage $\lambda=488, d)$ carrier concentration, $\lambda=488$. 
According to literature $[8,4]$, the $G$ peak position shifts to higher energy for increasing doping, no matter the sign of the charges (see Fig. 4). In our doped samples, an applied positive gate voltage causes an increase in doping and a shift in $G$ peak position to higher energy. An applied negative gate voltage results in a decrease in doping and a shift in $G$ peak position to lower energy. Even though these results at a first glance seem contradictory to theory, they are still in agreement with [8].Since the samples are doped; the charge neutrality point is far away from zero gate voltage. That means we have to apply a very large voltage to observe the trend reported in [8]. However, at high voltage the devices become unstable producing large hysteresis or they actually break .So, because the sample is doped, the graphs don't have the expected V-shape behavior, we are only probing half of it.

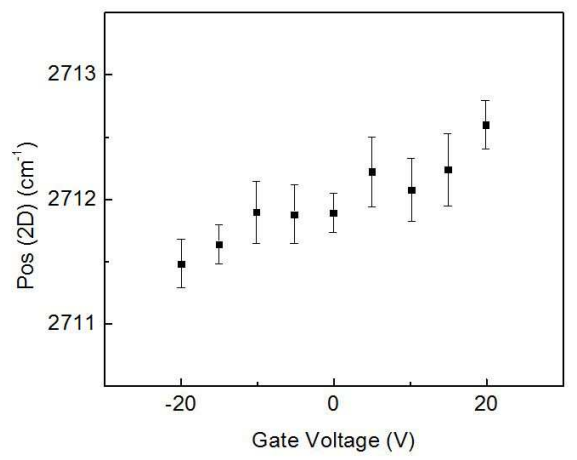

Fig. 5. $\operatorname{POS}(2 \mathrm{D})$ under apply gate voltage $(-20 \mathrm{~V},+20 \mathrm{~V})$ in monolayer graphene, $\lambda=488 \mathrm{~nm}$.

Fig 5 shows POS(2D)as a function of gate voltage. We do not notice any significant shifts, if we consider the resolution of our Raman setup. This is in agreement with existing literature, since our back gating approach only allows concentration slower than $1013 \mathrm{~cm}^{-1}$. This concentration is not enough to observe changes in POS (2D).

\subsection{Gated Bilayer graphene}

Bilayer graphene has a tunable band gap. When we apply gate voltage, the Fermi level will be shifted and we can observe POS $(\mathrm{G})$ changing. Our experimental results are consistent with theory. Fig 6 shows the Raman spectra taken at different values of $\mathrm{V}_{\mathrm{g}}$. We observe that both the position and the shape of the $\mathrm{G}$ band depend on $\mathrm{V}_{\mathrm{g}}$, in agreement with previous Raman studies of gated bilayer graphene [9, 1]. P.R. Wallace showed that the E-k relation is the following, $E=\hbar v F\left(k_{x}{ }^{2}+k_{y}{ }^{2}\right)^{1 / 2}$, where the Fermi velocity is $v_{F} \sim 10^{6} \mathrm{~m} / \mathrm{s}$ near the six corners of the hexagonal Brillouin Zone for low energies [9]. Due to the linear dispersion relation, electrons and holes in these six points behave as relativistic particles that can be described by the Dirac equation[9].Therefore, they are called Dirac fermions and these corners are called Dirac Points. The Dirac electrons can be controlled by application of external electric and magnetic fields. As we know $\varepsilon$ is directly relate to the position of the Fermi surface, $\varepsilon=\left|E_{F}\right|$, $\left|E_{F}\right|$ is the absolute value of the Fermi energy with respect to the Dirac point .In our experiments we were not able to observe the initial softening of the $\mathrm{G}$ band for $\varepsilon F<\hbar \omega G / 2$, as reported by Yan et al. [9]. This result can be explained by the presence of a nonhomogeneous charge distribution in our sample in the submicron range and by the fact that our experiment was done at room temperature [11]

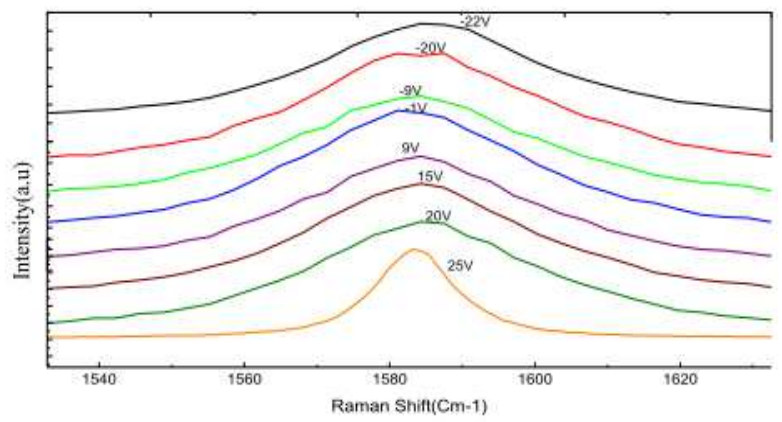

Fig. 6. G peak Raman spectra at different applied gate voltage.

Fig 7 shows the 2D peak shifts of pristine and transferred graphene as a function of carrier concentration. The $G$ peak and $2 \mathrm{D}$ peak positions increase in energy for increase doping concentration (applying gate voltage), no matter the sign of the charges.
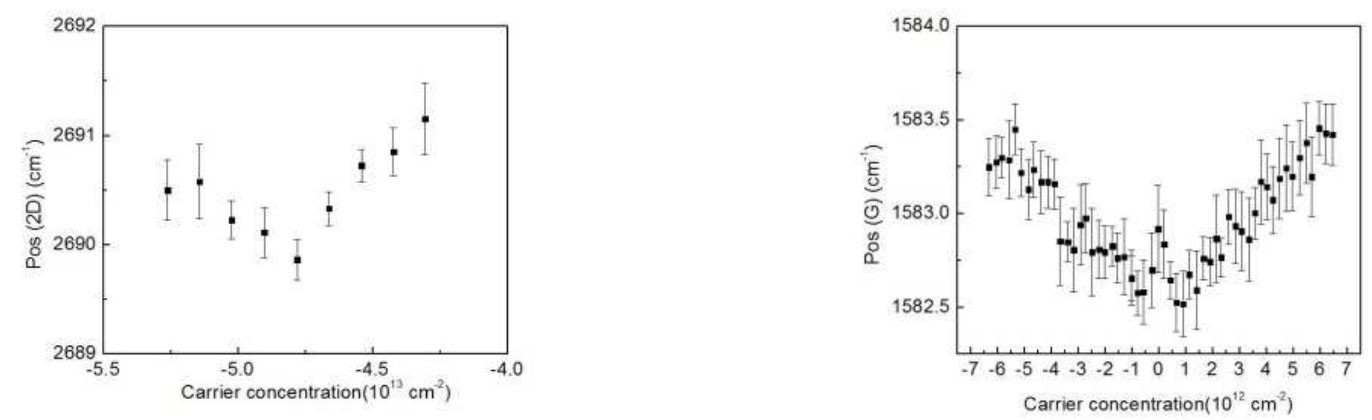

Fig. 7. POS (2D) as a function of carrier concentration: bilayer pristine graphene, $\lambda=514 n m$ (left) bilayer transferred graphene $\lambda=514 n m$, (applying gate voltage $(-30 \mathrm{~V},+30 \mathrm{~V})$ Step: $1 \mathrm{~V})$ (Right). 
The electronic structure of graphene changes when moving from monolayer to bilayer, three layers and so on up to about 10 layers. By applying positive or negative gate voltage, we will have positive or negative carrier concentration and we observe that the $G$ peak position increase in energy for increasing concentration, in agreement with theory, the opening of the band gap in bilayer graphene is based on the application of an electric field $\mathrm{E}$ perpendicular to the layers, given by:

$$
\mathrm{E}=\left(\mathrm{n}_{\text {top }}+\mathrm{n}_{\text {bot }}\right)|\mathrm{e}| /\left(2 \varepsilon_{0}\right)
$$

$\mathrm{n}_{\text {top }}$ are the charge carriers coming from the top and $\mathrm{n}_{\text {bot }}$ the charge carriers coming from the bottom of bilayer graphene, 0 is the vacuum permittivity, and $\varepsilon_{0}$ is the electronic charge[10] so changing in carrier concentration means changing in electric field and it cause changing in the position of the 2D peak and $\mathrm{G}$ peak. Fig 7, shows that pristine bilayer graphene and transferred bilayer graphene have different carrier concentrations $(n=C(V-V o))$, i.e. different $\mathrm{V}_{0}$.

\section{Conclusion and Future Work}

In conclusion, we have studied the Raman spectrum of mono and bi-layer graphene as a function of gate voltage. Raman spectroscopy is particularly useful for characterizing flakes since it is a fast, non-destructive method. By applying gate voltage to graphene and its bilayer, we shift the position of the Fermi level and tune the carrier concentration. These changes appear as shifts in the G and 2D peaks and can be used to extract the carrier concentration. Our experimental results are in agreement with Ando calculations [12].

Graphene research is still in its infancy. The work can be extended by calculating the carrier concentration on the top and bottom layer separately. The two will have different values (as expected) since the doping from the bottom layer will largely be due to the substrate, while doping for the top layer is due to expose in the atmosphere, for example, water adsorption, in addition, the experiment part can be down in different temperature especially high temperature results will be interesting. The investigation can follow by working on folded graphene of other few layer graphene.

\section{Acknowledgements}

This work could not be done without the help given to us by the people at the experimental physics department at freie
University of Berlin. We want to express special thanks to Dr. Cinzia Casiraghi, Prof. Håkan Pettersson, Prof. Donald Lupo, Dr. Hossein Gholizadeh, Dr. Axel Eckmann.

\section{References}

[1] Antonio.H. Castro Neto, 'The carbon new age',Materialstoday volume 13,2010 .

[2] C. Casiraghi, S. Pisana, k. S. Novoselov, A. K. Geim and A. C. Ferrari, "Raman fingerprint of charged impurities in graphene" ,Applied physics letters 91, 233108,2007.

[3] C. Cariraghi, A. Hartschuh, E. Lidorikis, H. Qian, H. Harutynyan, T. Gokus, K. S. novoselov and A.C. Ferrari, Reyligh imaging of graphene and graphene layers, Nano letters, volum7, no9, 2711-2717, 2007.

[4] Michele Lazzeri and Francesco Mauri, Nonadiabatic Kohn Anomaly in a Doped Graphene Monolayer, Physical Review Letters, 97(26):29, 32, 2006.

[5] Gregory F Schneider, Victor E Calado, HennyZandbergen, Lieven M K Vandersypen, and Cees Dekker, 'Wedging transfer of nanostructures' Nano letters, 10(5):1912,2010.

[6] CaglarO.Girit and A. Zettl, "Soldering to a single atomic layer", Applied physics letter 91, 2007.

[7] Paola Gava, Michele Lazzeri, A. Marco and FrancescoMauri, "Probing the elevtrostatic environment of bilayer graphene using Raman spectra", Physycal review B80, $155422,2009$.

[8] A.Das, S. Pisana, B. Chakaraborty, S. Piscanec, S. K. Saha, U. V. Waghmare, K. S. Novoselov, H. R. Krishnamurthy, A. K. Geim, A. C. Ferrari and A. K. Sood, "Monitoring dopants by Raman scattering in an electrochemically top-gated graphene transistor", Nano Letters 67,2008 .

[9] J. Yan et al, , Electric Field Effect Tuning of Electron-Phonon Coupling in Graphene,. Phys. Rev. Lett 101, 136804 ,2008.

[10] D.L. Mafra,P. Gava,L.M. Malard,R.S. Borges, G.G. Silva,J.A Leon,F. Plentz,F. Mauri,M.A. Pimenta, Characterizing intrinsic charges in top gated bilayer graphene device by Raman spectroscopy,Carbon 50,2012

[11] A. Das, B. Chakraborty, S. Piscanec, S. Pisana, A. Sood and C. Ferrari, "Observation of distinct electron-phonon coupling in gated bilayer graphene, Physical Review 101,257401, 2008.

[12] A. Das, B. Chakraborty, S. Piscanec, S. Pisana, A. k. Sood and A. C. Ferrari, "Phonon renormalization indoped bilayer graphene", Physical review B 79,155417, 2009. 